\title{
Evolution of Scale-Free Wireless Sensor Networks with Feature of Small-World Networks
}

\author{
Ying Duan, ${ }^{1,2}$ Xiuwen Fu, ${ }^{3}$ Wenfeng Li, ${ }^{1}$ Yu Zhang, ${ }^{1}$ and Giancarlo Fortino ${ }^{4}$ \\ ${ }^{1}$ School of Logistics Engineering, Wuhan University of Technology, Wuhan 430063, China \\ ${ }^{2}$ Department of Computer Science and Application, Zhengzhou University of Aeronautics, Zhengzhou 450015, China \\ ${ }^{3}$ Institute of Logistics Science and Engineering, Shanghai Maritime University, Shanghai 201306, China \\ ${ }^{4}$ Department of Informatics, Modelling, Electronics and Systems, University of Calabria, Via P. Bucci, Cubo 41C, 87036 Rende, Italy \\ Correspondence should be addressed to Xiuwen Fu; fuxiuwen1987@163.com and Wenfeng Li; liwf_cn@126.com
}

Received 19 April 2017; Accepted 28 June 2017; Published 30 July 2017

Academic Editor: Fabrizio Messina

Copyright (c) 2017 Ying Duan et al. This is an open access article distributed under the Creative Commons Attribution License, which permits unrestricted use, distribution, and reproduction in any medium, provided the original work is properly cited.

\begin{abstract}
Scale-free network and small-world network are the most impacting discoveries in the complex networks theories and have already been successfully proved to be highly effective in improving topology structures of wireless sensor networks. However, currently both theories are not jointly applied to have further improvements in the generation of WSN topologies. Therefore, this paper proposes a cluster-structured evolution model of WSNs considering the characteristics of both networks. With introduction of energy sensitivity and maximum limitation of degrees that a cluster head could have, the performance of our model can be ensured. In order to give an overall assessment of lifting effects of shortcuts, four placement schemes of shortcuts are analyzed. The characteristics of small-world network and scale-free network of our model are proved via theoretical derivation and simulations. Besides, we find that, by introducing shortcuts into scale-free wireless sensor network, the performance of the network can be improved concerning energy-saving and invulnerability, and we discover that the schemes constructing shortcuts between cluster heads and the sink node have better promoted effects than the scheme building shortcuts between pairs of cluster heads, and the schemes based on the preferential principle are superior to the schemes based on the random principle.
\end{abstract}

\section{Introduction}

Wireless sensor networks (WSNs) are usually made up of hundreds even thousands of distributed sensor nodes organized in ad hoc paradigm to monitor environments. Since they can be easily deployed and self-organized, WSNs can cover a wide range of applications domains [1,2]. As in most of scenarios WSNs are expected to operate in unattended environments, the sensor nodes always suffer from the risk of energy depletion, hardware malfunction, or deliberate attacks $[3,4]$. The failure of sensor nodes would split originally connected network topology, would reduce the coverage of the network, and might even lead to a global network paralysis [5]. So how to establish a stable and highly efficient WSNs topology has been a hot research issue in recent years.

Since the end of the 20th century, complex networks have become an important analytical tool and a research method in many interdisciplinary fields, such as the World Wide
Web, social networks, and scientific cooperation networks $[6,7]$. The discoveries of scale-free network and small-world network greatly promote the development of the complex networks research.

Barabási and Albert [8] firstly discover the scale-free feature in complex networks through analysis of degree portion of World Wide Web. They find that some nodes, which they called "hubs," have many more connections than others and that the network as a whole follow a power-law distribution of the number of links connecting to a node. They define this characteristic as scale-free and propose an evolution network model called Barabási-Albert (B-A) to generate scale-free structures. Through further analysis, many real networks like social and biological networks also present scale-free feature $[9,10]$. The most evident feature of scalefree network is that the degree distribution of the network $P(k)$ is in line with power-law distribution. As in scalefree networks the high-degree nodes only account for small 
proportion of the network, the failure probability of these nodes is at a relatively low level when facing random failure. By contrast, as for the low-degree nodes that widely spread in the network, although these nodes have to take much a higher failure risk, the impact of these node failures has little effect on network performance. Due to this reason, scale-free networks demonstrate excellent survivability against random attacks. And there has already been considerable interest in construction of a highly survivable network topology by introducing scale-free feature. As WSNs are a particular type of complex networks, scale-free networks and related theories also provide us with a new inspiration to establish a highly error-tolerant WSNs topology [11]. However, since in WSNs high-degree nodes are always required to relay most of data flow to the base station, the energy of these nodes would be depleted rapidly so that normal operation of the entire network is threatened. Thus, improving the network energy balance and energy consumption efficiency is the major challenge before establishing a highly efficient and reliable scale-free WSNs topology.

As a transitive network type between random network and regular network, small-world network was proposed in 1998 by Watts and Strogatz [12]. Although most of nodes in small-world network are not directly connected, the vast majority of nodes can be connected to each other only via a few hops. It means that the small-world network exhibits a small average shortest path length along with a big clustering coefficient. As far as WSNs are concerned, lowering relay hops from sensor nodes to the base station is the basic idea to improve the network performance in terms of lifetime, overhead, and delivery delay. Thus, it is reasonable to expect that constructing WSNs topology with small-world effect is a feasible method to reduce the network energy consumption and improve the network connectivity. But obviously, as WSNs usually consist of a large number of sensor nodes, how to design a cost-effective strategy to build small-world WSNs is an extremely challenging research direction.

Although the excellent topological properties of scalefree and small-world effects offer potential ways to improve the network performance of WSNs, either of them can only cover one or two aspects of the network performance; that is, scale-free effect focuses on the enhancement of network survivability and small-world effect attempts to shorten the transmission paths within the network. So the network topology obtained by only using scale-free or small-world effect is still far from satisfactory. Due to this reason, it is important to investigate whether or not it is possible to build WSN topologies by both considering scale-free and smallworld effects, thus making the network highly error-tolerant as well as maintaining reasonable energy efficiency. Aiming to tackle this issue, in this paper we propose a new topology evolution model for WSNs by considering scale-free and small-world at the same time. The evolution model has the following characteristics: (1) by introducing cluster heads and cluster members into the network according to the predefined cluster ratio, the generated network topology is featured by cluster structure which is more closed to application cases of WSNs; (2) to reduce the energy consumption and prolong the lifetime of the network, in our mechanism the cluster heads with high-degree and sufficient energy are more likely to have new connections and the maximum number of connections a cluster head could possess is constrained by the degree saturation; (3) aimed at further improving the energy balance and energy consumption efficiency in generated scale-free WSNs topology, shortcuts are introduced into the network during the evolution process to achieve small-world effect. Four shortcut deployment schemes (i.e., RC, SC, RCS, and SCS) are presented; (4) it has been proven that building shortcuts between schemed clusters (i.e., cluster heads with highest degree) and the sink is the most effective way to maximize the lifting effects of shortcuts among four proposed deployment schemes.

The contribution of this paper is twofold:

(1) An evolution model of scale-free WSN with feature of small-world networks is proposed. The generated topology by this model, on the one hand, can have high error tolerance due to the scale-free feature; on the other hand the network performance can also be guaranteed in terms of energy balance and energy consumption due to the small-world effect. To the best of our knowledge, this is the first evolution model that combines characteristics of scale-free network and small-world network together.

(2) Aimed at fully investigating the lifting effects of shortcuts, four shortcuts deployment schemes are designed. Through experiments, we discover that the schemes that construct shortcuts between cluster heads and the sink node have better promoted effects than the scheme that builds shortcuts between pairs of cluster heads. The schemes based on the preferential principle (i.e., more prone to connect with highdegree cluster heads) are superior to the schemes based on the random principle (i.e., selecting the cluster heads to build connections with randomly).

The remainder of this paper is organized as follows. In Section 2, the related researches of scale-free network and small-world network in WSNs are introduced. Section 3 presents our evolution model and carries out a simple analysis of the model. In Section 4, theoretical analysis is made to verify the scale-free effect of our model by using mean-field theory. In Section 5, simulations and analysis are conducted to evaluate the network performance of our model. Finally, conclusions and future works are presented in Section 6.

\section{Related Work}

2.1. Scale-Free Networks. As scale-free networks are robust against random removal or failures of nodes, this inspires us to construct a high survivability network for WSNs by using scale-free theory. And there have already been many researches around this direction. As far as we know, Chen et al. [13] is the first proposal aiming at applying scalefree theory to the topology construction of WSNs. In this research, authors assume that when a new sensor node joins the network, it firstly selects a certain number of existing sensor nodes randomly from the network to form an optional 
nodes set which we usually refer to as "local-world area" and on the basis of principle of "preferential attachment" in B-A model [9], the newly coming node can only connect to the node that belongs to its local world and has higher probability to attach to the node having higher degree. Although in the research of Chen et al. the scale-free property of their model has been successfully proven, the energy sensitivity of WSNs is not taken into account. Zhu et al. [14] proposed two scale-free evolution models of WSNs: EAEM (Energy-Aware Evolution Model) and EBEM (Energy-Balanced Evolution Model). In EAEM, the newly joining nodes are given higher priority to connect to the node with higher residual energy. In EBEM, besides the energy factor, the connectivity degree is also an impact factor for newly joining nodes to choose existing nodes to connect to which. The experiments suggest that the performance of EBEM is superior to that of EAEM in terms of energy-saving. Moreover, it is worth noting that, in order to avoid one sensor node having excessive connections, EBEM introduces degree saturation constraint, the purpose of which is to set an upper limitation for the connections the node could have. In Chen et al. and Zhu et al., authors made the simple assumption that WSNs are homogeneous, in which the role and functions of the nodes are totally the same.

But actually in most real scenarios, WSNs are clusterstructured, in which the nodes can be subcategorized into regular nodes and cluster heads. Regular nodes are responsible for sensing environment and centralizing the sampling data into their cluster heads. Cluster heads are in charge of relaying data to the base station. Based on this consideration, Li et al. [15] proposed a heterogeneous evolution model of WSNs. In such model, the role of the newly joining sensor node (i.e., regular sensor node or cluster head) is predetermined by setting the parameter "cluster head ratio (i.e., the proportion of the cluster heads in the network)" before it joins the network. And the joining node can only be linked to the existing cluster heads. Through this mechanism, if the role of the newly joining node is set as regular node, its degree can only be one. By contrast, if the newly joining node is determined as cluster head, it is possible to obtain more connections. Building on this result, Jiang et al. [16] investigated an improved heterogeneous evolution model based on localworld (L-W) model. Compared with B-A model that selects connected objects out of the global network, the new nodes can only build connections with the objects from the local world (i.e., defined area). Since the scale of local-world area is adjustable, the power-law exponent of the obtained model can be adjusted according to the needs of the applications.

In the above studies, the connecting node is selected by considering connectivity degree and residual energy. However, the topology of the WSNs is also constrained by the transmitting power of the sensor nodes. Obviously, aiming to conserve energy, most of sensor nodes are not necessarily required to maintain maximal transmitting power. Due to this reason, Qi et al. [17] introduced adaptable communication range into the topology control algorithm. Through this way, the topology generated by Qi et al. follows the feature of being scale-free as well as maintaining low-level energy consumption. In the real environment, besides node addition, node deletion and link reconstruction are also possible to occur during the evolution process. For this reason, Zheng et al. [18] proposed an evolution model of WSN with reconstruction mechanism. As the dynamic behavior of nodes and links is more diversified in Zheng's model, the generated topology is more flexible and accommodating in practice. Most of the existing evolution models are derived from B-A model or L-W model. Some researchers attempted to build scale-free topology of WSNs by using randomwalk (R-W) model $[19,20]$. Differently from the principle of preferential attachment in B-A model and L-W model, $\mathrm{R}-\mathrm{W}$ model uses the times of the walker passing by to determine the probability of the existing nodes obtaining the new connections with new-joining nodes. Although WSN topologies created by using the R-W model are not so different from topologies constructed through B-A or L-W models, the R-W model still offers us new means of design.

2.2. Small-World Networks. By introducing some shortcuts (i.e., random connections) into the regular network, the network can have a small average shortest path length along with a big clustering coefficient. Obviously, in Internet or social networks, shortcuts can be easily built. But as for WSNs, building shortcuts is a challenging issue due to the physical limitations brought by limited wireless transmission radius. Helmy et al. [21, 22] firstly proved that small-world effect can also be applied in WSNs by introducing long-distance wired cables. In their solution, a certain amount of wired cables is deployed in the network and the cables can make the sensor nodes at their ends communicate with each other. Since the length of the deployed cables could be longer than the wireless transmitting radius of the sensor node, wired cables can play the same role of shortcuts. Sharma and Mazumdar [23] carried out a further research about the effects of shortcuts. As for the WSN made up of 1000 uniformly distributed nodes, only 5-24 shortcuts are required; the average path of the network can be shortened by $60 \%-70 \%$. Hawick and James [24] researched the lifting effects of shortcuts from the perspective of the coverage, error tolerance, and energy conservation. The research result suggests that the introduction of the small-world network features into WSNs can substantially reduce the probability of isolated clusters appearing in the network and then sufficiently improve the whole coverage effect and the lifetime of WSNs.

Although in [20-25] the advantage of small-world WSN has been proven, the effects of shortcuts fail to achieve optimal performance as the shortcuts are randomly deployed. Aiming to improve the lifting effects of shortcuts, Guidoni et al. [26] proposed two shortcuts placement schemes: DAS (Directed Angulation towards the Sink) and SSD (Sink Node as Source/Destination). In DAS, the ends of the shortcut are sensor nodes and the directions of shortcuts are towards the sink node. In SSD, one side of the shortcut is sensor nodes, while the other side is the sink node. Simulation results demonstrate that SSD outperforms DAS in terms of latency and energy conservation. Although SSD is superior, DAS is a more realistic plan as the sink node cannot provide sufficient interfaces for SSD in most cases. Since the premise of implementing DAS is that each sensor node needs to have the location knowledge of all nodes in the network, it might 
pose a huge issue for practical implementation. On the basis of DAS, Guidoni et al. [27] proposed an improved version ODASM (Online Directed Angulation towards the Sink node Model). In ODASM, the nodes are only required to know the geographical location of the nodes within their communication range. Although Guidoni's solutions have already been proven to be more effective than random deployment scheme, there is still a certain extent of randomness in placement of shortcuts. Due to this reason, we designed a deterministic placement scheme for shortcuts in WSN [28]. In our scheme, we firstly used WSN-betweenness centrality to locate the nodes with heavy traffic task and build the shortcuts to lower the centrality of these nodes, thus improving the energy balance level of the network. Compared with DAS and SSD, the network life can be prolonged by $50 \%$. But our scheme presented in [28] cannot operate in a decentralized way as the access of WSN-based betweenness centrality relies on the topology information of the entire network.

From the above analysis, we can easily find that although some promising progress about scale-free network and smallworld network has been made, currently both theories are not jointly applied to have further improvements in the generation of WSN topologies. Therefore, we propose a clusterstructured evolution model of WSNs considering the characteristics of both scale-free network and small-world network to improve the network performance in terms of error tolerance and energy efficiency. To the best of our knowledge, this is the first evolution model that combines characteristics of scale-free network and small-world network together.

\section{Evolution Model}

3.1. Model Assumption. As WSNs are energy-sensitive networks, how to prolong the lifetime of the network is always a central topic in the study of WSNs. Aiming to achieve this goal, in most cases, clustering structure is introduced into the network to guarantee cost-effective data transmission via multihops [29]. Thus, in our model, firstly we set that the choice made by newly joining node for selecting connected objects is affected by the connectivity degree and residual energy that the existing node has. In other words, if the existing cluster head has more connections and more energy, it will more likely build links with new incoming nodes. Secondly, in order to avoid the overconsumption of the energy, we set an upper limit for the number of connections that a cluster head could have; here we call this degree limit degree saturation $k_{\max }$. Finally, in each time step, the existing network topology could have a shortcut. Before presenting our model, we need to note that in WSNs the energy of each sensor node is consumed in different way and the nodes spend their most energy in data transmission after the networks have already been organized, so we assume that the remaining energy of the nodes is fixed during the evolution process. And because of the constraint of node transmission radius, each sensor node in the network can only communicate with those nodes within its transmission range. For clearance, we list some important parameters used to characterize the wireless sensor networks in Notations.
3.2. Design of Evolution Model. The generation of a network is as follows.

(1) Initialization. Starting from $m_{0}$ cluster heads and $e_{0}$ edges, initialized network includes a sink node/base station. To ensure no isolated nodes exist, each cluster node at least has an effective path towards the sink node.

(2) Preferential Attachment. At every time step, a new cluster head or regular node with one edge enters into the existing network with probability $p$ or $1-p$, respectively. A small number of cluster heads would make lots of regular sensor nodes connect to them, which results in heavy operating burden and consuming their energy fast, but large number of cluster heads would waste the network resource and raise the hardware costs. Therefore, it is better for us to set $p$ between 0.1 and 0.5 . For convenience, we denote the newly coming node as node $j$. And then, the new node $n$ is given an energy value $E_{j}$ that obeys a specific probability distribution. When a new node $j$ comes into the network, it would only choose one cluster head $i$ from existing network topology to build connection with. The new node $j$ links to the cluster head $i$ according to the probability $P_{s}(i)$

$$
P_{s}(i)=\left(1-\frac{k_{i}}{k_{\max }}\right) \frac{E_{i} k_{i}}{\sum_{j=1}^{N(t)} E_{j} k_{j}},
$$

where $N(t)$ is the sum of cluster heads at the existing moment $t$. It is easy to find that the bigger $E_{i}$ and $k_{i}$ are, the more possibility the cluster head $i$ can have to connect to the new node $n$. But if the degree (number of connections) of cluster head $i$ reaches the degree saturation $k_{\max }, 1-k_{i} / k_{\max }$ becomes zero and $P_{s}(i)$ is also equal to zero, which means the cluster head $i$ cannot have more connections if its degree achieves $k_{\text {max }}$. Through this mechanism, we can ensure that the cluster heads with high-degree and sufficient energy are more likely to have new connections while their energy-consuming speed can also be controlled within a reasonable scale.

(3) Adding of Shortcuts. At each time step, a shortcut is added to the current network with probability $q$. To accurately evaluate the lifting effects of shortcut on network performance, four types of placement schemes of shortcuts are given.

Scheme RC (Random Clusters). Two cluster heads are randomly chosen from the existing network to build a shortcut.

Scheme SC (Schemed Clusters). Two cluster heads are chosen to build a shortcut based on their connectivity degree and residual energy. If the cluster head has ampler energy and more degrees, it has more priority to be chosen as the endpoint of the shortcut. The concrete steps of the scheme SC are as follows: first of all, a cluster head $i$ is selected from the current network as the starting point of the shortcut according to the probability $P_{a}(i)$. And then, the ending point of the shortcut is chosen from the rest of the cluster heads in the network according to the probability $P_{a}(i)$

$$
P_{a}(i)=\frac{E_{i} k_{i} / w_{i}}{\sum_{j=1}^{N(t)} E_{j} k_{j} / w_{j}},
$$




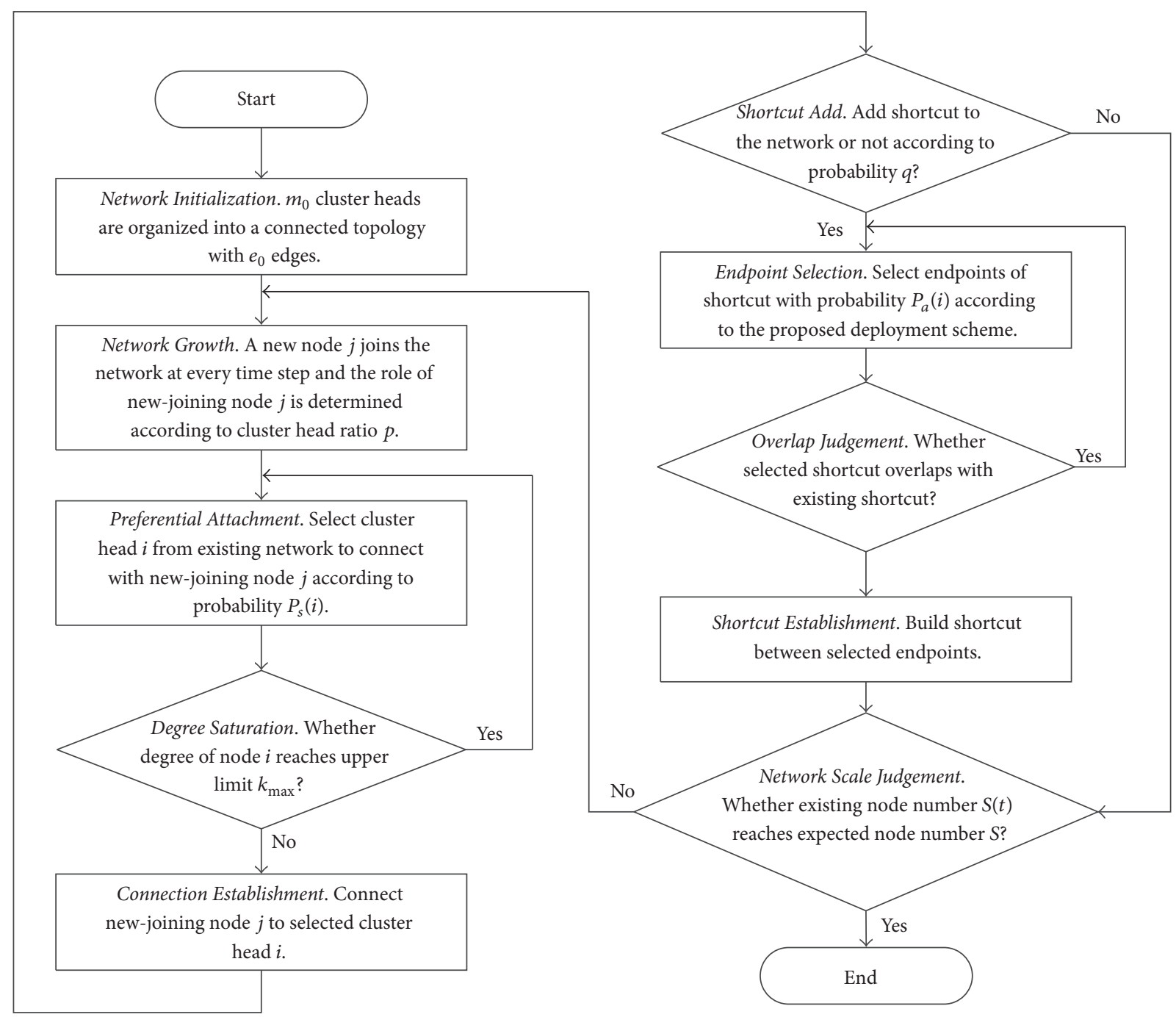

FIgURE 1: Flow chart of our model.

where $N(t)$ and $w_{i}$ are the sum of cluster heads and the number of shortcuts the cluster head $i$ has, respectively. In order to avoid too many shortcuts overconcentrating on a few of highly central cluster heads, we assume that the more shortcuts the cluster head has, the lower the probability of it obtaining next shortcut. Thus, we introduce $w_{i}$ into $P_{a}(i)$ to achieve this.

Scheme RCS (Random Cluster towards Sink). One cluster head is randomly selected from the network and the shortcut is built between the selected node and the sink node.

Scheme SCS (Schemed Cluster towards Sink). One cluster head is selected from the network with the probability $P_{a}(i)$

$$
P_{a}(i)=\frac{E_{i} k_{i}}{\sum_{j=1}^{N(t)} E_{j} k_{j}} .
$$

And then, a shortcut is built from the selected cluster head to the sink node.
Here, we still need to ensure that if the shortcut we build overlaps with the existing links in the network, the shortcut should be rebuilt until it does not overlap with the existing links. In order to state that more clearly, Figure 1 presents the flow chart of our model.

3.3. Example. Figure 2 depicts the topologies generated by our model with four adding schemes of shortcuts. The generated topology consists of 100 sensor nodes and the sink node is by default located at the center of the area. The simulation area is $100 \mathrm{~m} \times 100 \mathrm{~m}$. The transmission radius of sensor node is $20 \mathrm{~m}$. Adding probability of shortcuts $q$ is 0.1 , ratio of cluster head $p=0.2$, and degree saturation $k_{\max }$ is set to 20 . By referencing the energy configuration in [13], the remaining energy of nodes in our model obeys normal distribution $N(2,1)$. Regardless of shortcuts, the average degree of the generated network $\langle k\rangle=1.8$. The degree distribution of the networks exhibits tangible characteristic of being scale-free: the degree of most of nodes (no matter whether cluster heads or regular sensor nodes) is one and only a few of cluster 


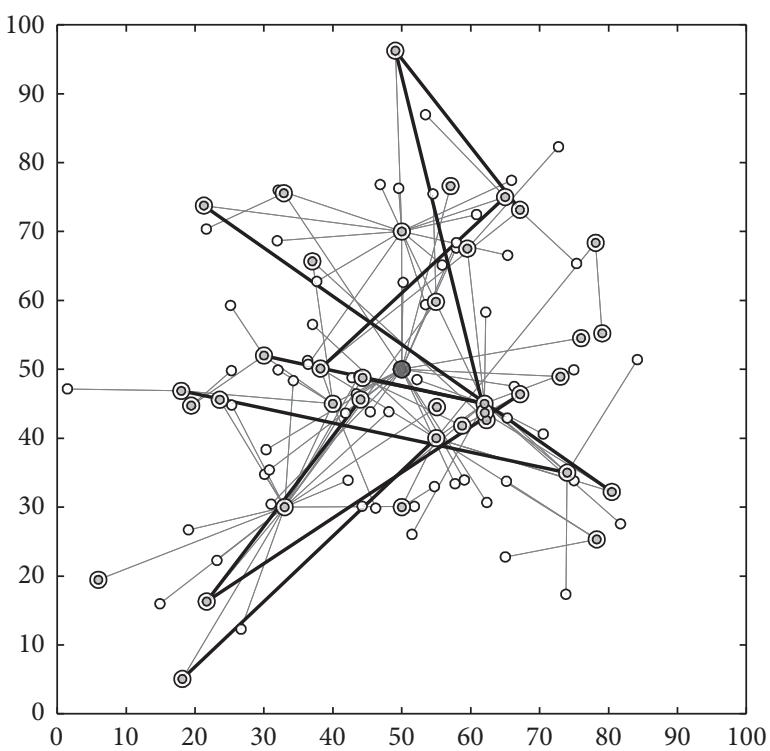

(a)

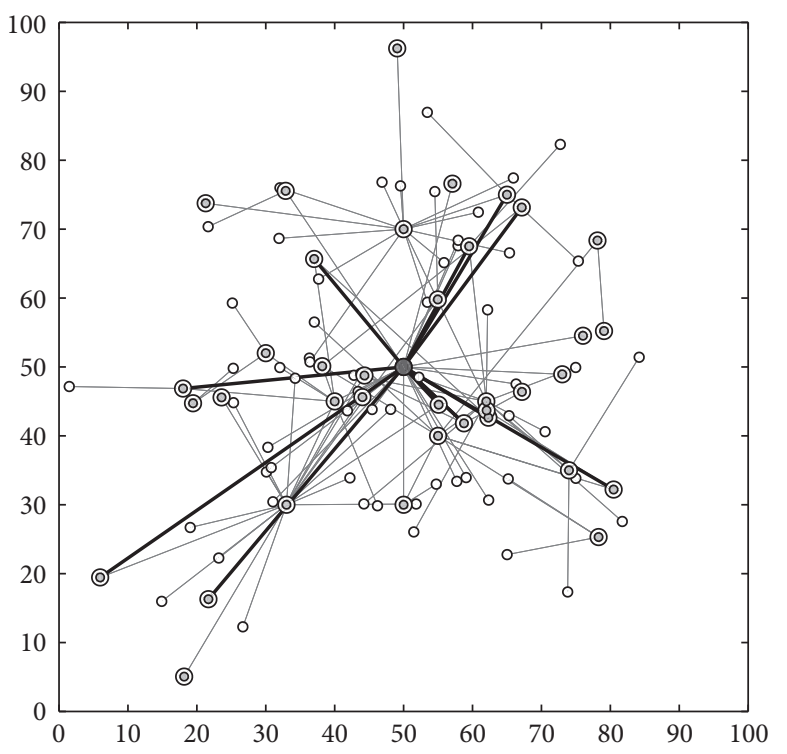

(c)

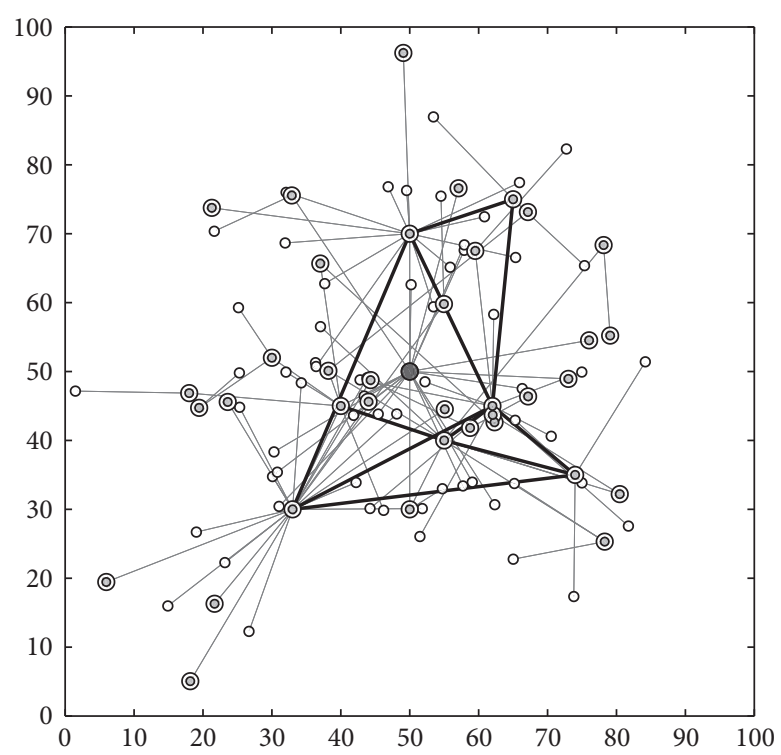

(b)

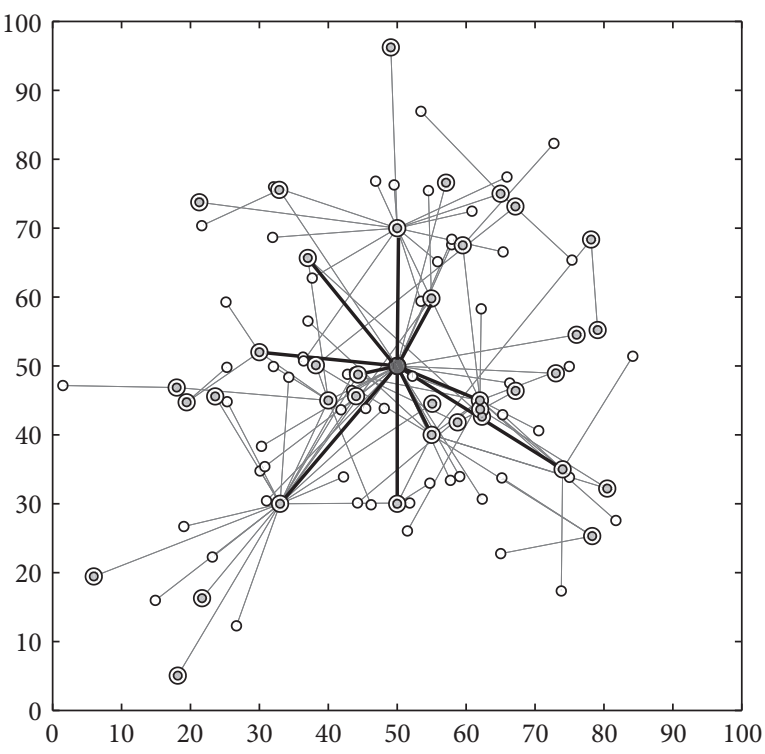

(d)

FIGURE 2: Scale-free WSNs topology under different adding schemes of shortcuts. (a) Scheme RC. (b) Scheme SC. (c) Scheme RCS. (d) Scheme SCS.

heads (less than three) reach degree saturation $k_{\max }=20$. It is easy to find that the shortcut deployments based on four schemes demonstrate evident differences. In Figure 2(a), with adding shortcuts based on scheme RC, the spreading feature of shortcuts complies with the characteristics of random distribution. Most of shortcuts choose the "edge" endpoints of which degree is one. Obviously, since most of the nodes in the topology are the edge node, these nodes have much higher probability to be selected randomly. As shown in Figure 2(b) where the shortcuts are placed according to scheme SC, the shortcuts are generally placed between high-degree cluster heads. If we observe more carefully, we can find that the shortcuts compose roughly a "ring." This phenomenon can be explained from two aspects: (1) in scheme SC the endpoints of the shortcuts are selected according to the degree and residual energy; this decides that these "central" nodes are still much more likely to obtain new shortcuts than "edge" node even if their attractions to new shortcut would be weakened with the increasing number of shortcuts they already have; (2) due to the "avoid overconcentrating" mechanism, one central cluster head is not allowed to have too many shortcuts; the opportunity of "central" cluster heads getting new shortcuts is approaching similarity to the process of the evolution. Figure 2(c) describes the network topology generated by scheme RCS. Since the starting points of the shortcuts are randomly selected in scheme RCS, most of the shortcuts are built between "edge" cluster head and the sink node. As shown in Figure 2(d), by contrast with scheme RCS, the 
starting points of the shortcuts in scheme SCS are always "central" nodes. This is because the selection of the starting points in scheme SCS is according to residual energy and the number of connections the existing cluster head has.

\section{Theoretical Analysis}

Through the analysis of this section to verify whether the model has B-A characteristics, at present, degree distribution $P(k)$ is the probability that a randomly chosen node has $k$ connections (or neighbors) and it can also be defined as the fraction of nodes in the network with degree $k$. Since it is the most important property that characterizes a network structure, in this section we will carry out a theoretical analysis on degree distribution $P(k)$ of the network in our model. Nowadays, there have already been many methods that can be used to research degree distribution of the evolving network (e.g., mean-field theory [8], master equation method [30], and rate equation method [31]). By referencing the derivation process of B-A model, here in our model we choose meanfield theory. For clearance, the following parameters are defined in Notations.

Firstly we need to specify that since, in schemes RC and RCS, cluster heads are randomly chosen, we define the shortcuts-selecting probability $P_{a}(i)$ as

$$
P_{a}(i)=\frac{1}{N(t)} .
$$

Suppose $k_{i, t}$ is the degree of cluster head $i$ at time $t$. In step (2), the cluster head is connected to the new incoming node according to the preferential attachment probability $P_{s}(i)$ and, in step (3), the cluster heads are selected as endpoints of the shortcuts according to the probability $P_{a}(i)$. So in schemes RC and SC, the growth rate of $k_{i, t}$ can be described as (5) and in schemes RCS and SCS, the growth rate of $k_{i, t}$ can be described as (6):

$$
\begin{aligned}
& \frac{\partial k_{i, t}}{\partial t}=p P_{s}(i)+q P_{a}(i), \\
& \frac{\partial k_{i, t}}{\partial t}=p P_{s}(i)+2 q P_{a}(i) .
\end{aligned}
$$

In (5) and (6), the first item means the probability of cluster head $i$ connecting to new-coming node and the second item means the probability of cluster head $i$ being an endpoint of the new shortcut. In schemes RC and SC the shortcut is built between two selected cluster heads; cluster head $i$ can be double-selected when a new shortcut comes into the network. By contrast, for schemes RCS and SCS, the shortcut is between the selected cluster head and the sink node; cluster head $i$ can only be selected once. That is why in (6) the probability of cluster head $i$ being an endpoint of the new shortcut is twice the probability in (5). Given space limitation, we only analyze the degree distribution of the model with scheme RC. Equation (6) can be rewritten as

$$
\begin{aligned}
\frac{\partial k_{i, t}}{\partial t} & =p P_{s}(i)+2 q P_{a}(i) \\
& =\left(1-\frac{k_{i, t}}{k_{\max }}\right) \frac{E_{i} k_{i, t}}{\sum_{j=1}^{N(t)} E_{j} k_{j, t}}+\frac{2 q}{N(t)} .
\end{aligned}
$$

In our model, the degree $k_{i, t}$ of most of the cluster heads is much smaller than the degree saturation $k_{\max }$; we can obtain the following:

$$
1-\frac{k_{i, t}}{k_{\max }} \approx 1 .
$$

Since the network topology generated by our model is mainly applied in the scenario that requires large-scale deployment, we can reasonably assume that, after experiencing a longterm evolution, the number of sensor nodes has reached a size large enough. Thus, we can get the following:

$$
\begin{aligned}
N(t) & =m_{0}+p t \approx p t, \\
\sum_{j=1}^{N(t)} E_{j} k_{j, t} & =N(t)\langle k(t)\rangle \bar{E} \approx p t\langle k(t)\rangle \bar{E} \sum_{j=1}^{N(t)} E_{j} k_{j, t},
\end{aligned}
$$

where $t$ is the elapsed time, $\langle k(t)\rangle$ is the average degree at the time $t$, and $\bar{E}$ is the mean value of the residual energy of the nodes in the network. Substitute (8)-(9) into (7); then we can get

$$
\frac{\partial k_{i, t}}{\partial t}=\frac{E_{i} k_{i, t}}{t\langle k(t)\rangle \bar{E}}+\frac{2 q}{p t} .
$$

According to the method described by Sarshar and Roychowdhury [32], we can get the dynamic equation (11) of the total number of edges in the network

$$
\frac{d E(t)}{d t}=1+2 q
$$

Assuming $S(t)$ is the total degree of the network at time $t$, we can easily get

$$
E(t)=\frac{S(t)}{2} .
$$

Putting (12) into (11), we can get

$$
\frac{d S(t)}{d t}=2(1+2 q) \text {. }
$$

To solve (13), we can get

$$
S(t)=2(1+2 q) t .
$$

Only considering the scenario of long-term network evolving, we can get

$$
\langle k(t)\rangle=\frac{S(t)}{N(t)}=\frac{2(1+2 q) t}{p t}=\frac{2(1+2 q)}{p} .
$$


Putting (15) into (10),

$$
\frac{\partial k_{i, t}}{\partial t}=\frac{E_{i} k_{i, t} p}{2(1+2 q) \bar{E} t}+\frac{2 q}{p t} .
$$

Assuming $A=2 q / p$, (16) can be simplified as

$$
\frac{\partial k_{i, t}}{\partial t}=\frac{E_{i} k_{i, t}}{(2 / p+2 A) \bar{E} t}+\frac{A}{t} .
$$

Via equivalent transformation of (17), we can get

$$
\frac{\partial k_{i, t}}{E_{i} k_{i, t} p /(2+2 A p) \bar{E}+A}=\frac{\partial t}{t} .
$$

Concerning the generation rules of the network, each cluster head $i$ has initial degree $k_{i, t}=1+2 q$. Considering this as the initial conditions for (18), we can solve it and get

$$
k_{i, t}=\left(1+2 q+\frac{A}{Q}\right)\left(\frac{t}{t_{i}}\right)^{Q}-\frac{A}{Q},
$$

where $Q=E_{i} p /(2+2 A p) \bar{E}$. Equation (19) can be used to get the probability of $P\left(k_{i, t}<k\right)$

$$
\begin{aligned}
P\left(k_{i, t}<k\right) & =P\left\{t_{i}>t\left(\frac{k+A / Q}{1+2 q+A / Q}\right)^{-1 / Q}\right\} \\
& =1-P\left\{t_{i} \leq t\left(\frac{k+A / Q}{1+2 q+A / Q}\right)^{-1 / Q}\right\} .
\end{aligned}
$$

Generally, assume that sensor nodes are added to the network at regular intervals, so $t_{i}$ has equal probability density $P\left(t_{i}\right)$

$$
P\left(t_{i}\right)=\frac{1}{m_{0}+t} \text {. }
$$

So degree distribution of node $i$ will become

$$
P\left(k_{i, t}<k\right)=1-\frac{t}{m_{0}+t}\left(\frac{k+A / Q}{1+2 q+A / Q}\right)^{-1 / Q} .
$$

Considering energy $E_{i}$, degree distribution $P(k)$ of the network can be calculated as

$$
P(k)=\frac{\partial P\left(k_{i, t}<k\right)}{\partial k}=\frac{(k+A / Q)^{-1 / Q-1}}{Q(1+2 q+A / Q)^{-1 / Q}} .
$$

Concerning the energy-sensitivity property of the network, put $A=2 q / p$ and $Q=E_{i} p /(2+2 A p) \bar{E}$ into (23); the network degree distribution $P(k)$ is given as follows:

$$
\begin{aligned}
P(k) & =\int_{0}^{E_{\max }} \frac{(k+A / Q)^{-1 / Q-1}}{Q(1+2 q+A / Q)^{-1 / Q}} \rho(E) d E \\
& \propto \int_{0}^{E_{\max }} \frac{k^{-\gamma}}{Q(1+2 q+A / Q)^{-1 / Q}} \rho(E) d E,
\end{aligned}
$$

where $\rho(E)$ is the probability density of energy $E$ in the interval $\left[0, E_{\max }\right]$ and $E_{\max }$ is the maximum energy value a sensor node could possibly have. It is obvious that the network degree distribution $P(k)$ of our model is in line with the general form of the power-law distribution $P(k) \sim$ $k^{-\gamma}$ and the power-law exponent $\gamma=1+1 / Q . P(k)$ is closely associated with $\rho(E)$, the upper limit of energy $E_{\max }$, the proportion of cluster head nodes $p$, and probability of shortcuts $q$ but is unrelated to network scale, which is apparently featured by scale-free characteristics.

\section{Experiments}

In this section, through the simulations based on Matlab, we carry out further analysis on the network performance of our model from the following four perspectives: (1) confirming the small-world characteristics of our model by analyzing the network average path and clustering coefficient; (2) verifying the scale-free characteristics of our model through analysis of network degree distribution; (3) analyzing the network performance of our model in terms of energy consumption; (4) estimating the invulnerability of our model towards random attack. Aiming to have an accurate assessment of statistics characteristics and network performance of our model, the network scale is expanded reasonably. The specific simulation parameters are shown as follows: the simulation area is $200 \mathrm{~m}$ $\times 200 \mathrm{~m}$; the transmission radius of sensor nodes is $20 \mathrm{~m}$; the total number of nodes after final evolution $S=400$; the proportion of cluster heads $p=0.3$; the initial network includes $m_{0}=10$ cluster heads and $e_{0}=20$ edges and each cluster head can maintain at least one effective path towards the sink; the sink node is positioned at the geographical center of the simulation map; degree saturation $k_{\max }=50$ and initial energy of the node obeys normal distribution $N(2,1)$. Similar to most of realistic scenarios, we reasonably assume the sink node does not have energy constraint during the process of the simulation. When estimating the clustering coefficient, degree distribution, and average of the network, the impacts of the sink node are ignored. All the required data is the average value obtained by 50 times of simulations.

5.1. Analysis of Small-World Characteristics. As mentioned before, the most evident features of small-world networks are small average path length and big clustering coefficient. Therefore, we select average path length and clustering coefficient as metrics to verify whether or not the network topology generated by our model has small-world characteristics. But it is worth noting that, in the general complex networks, average path always denotes the average number of steps along the shortest paths for all possible pairs of network nodes, but for WSNs, since data delivery is directional which means all the collected data is converged at the sink node, the general definition of average path length does not suit WSNs. Therefore, we redefine the average path length in WSNs as the average number of steps along the shortest paths from each sensor node to the sink node.

Figure 3 shows the performance curve of normalized average path $L(q) / L(0)$ and clustering coefficient $C(q) / C(0)$ 


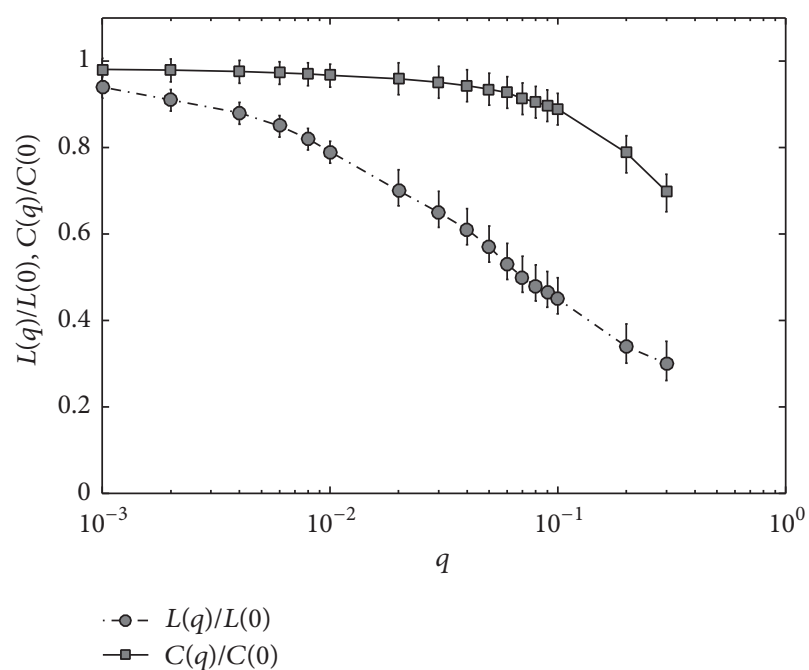

(a)

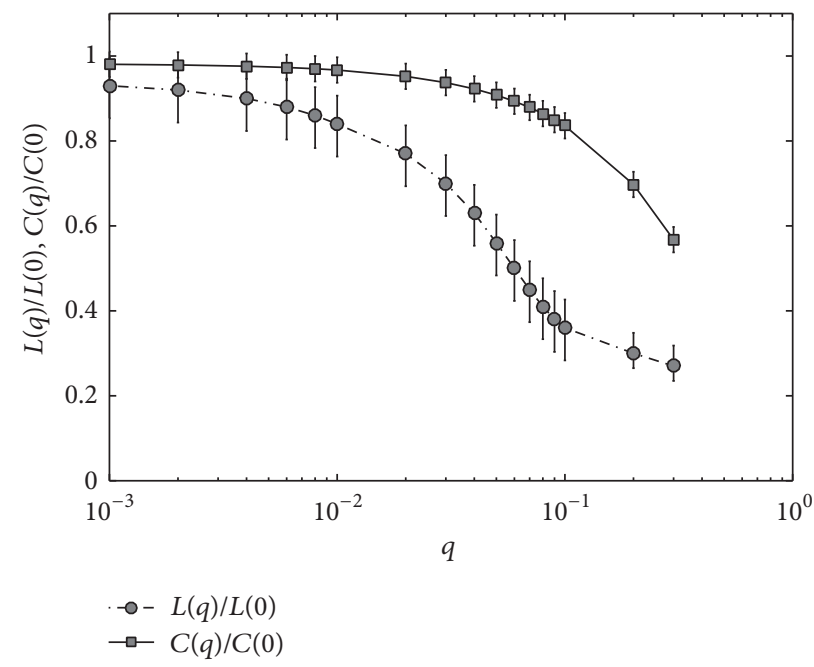

(c)

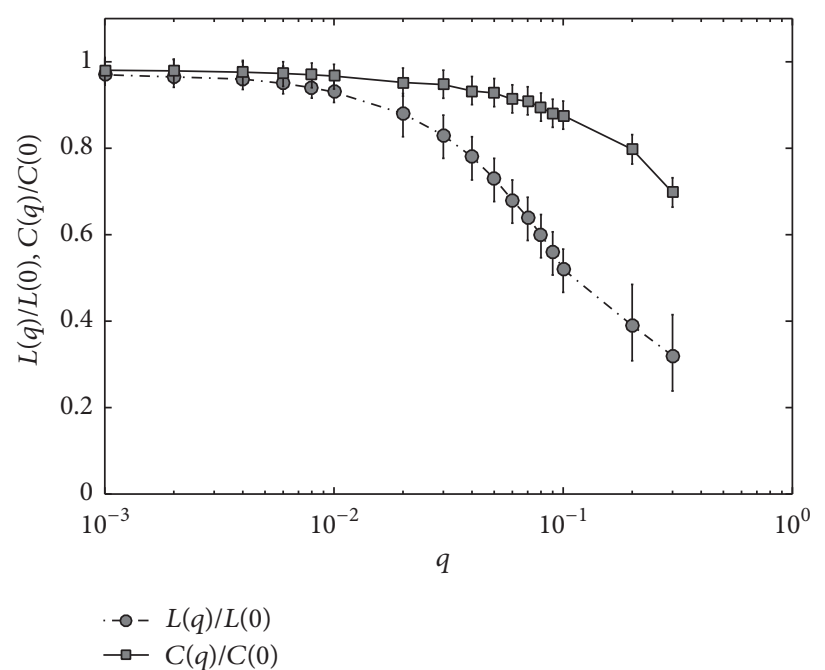

(b)

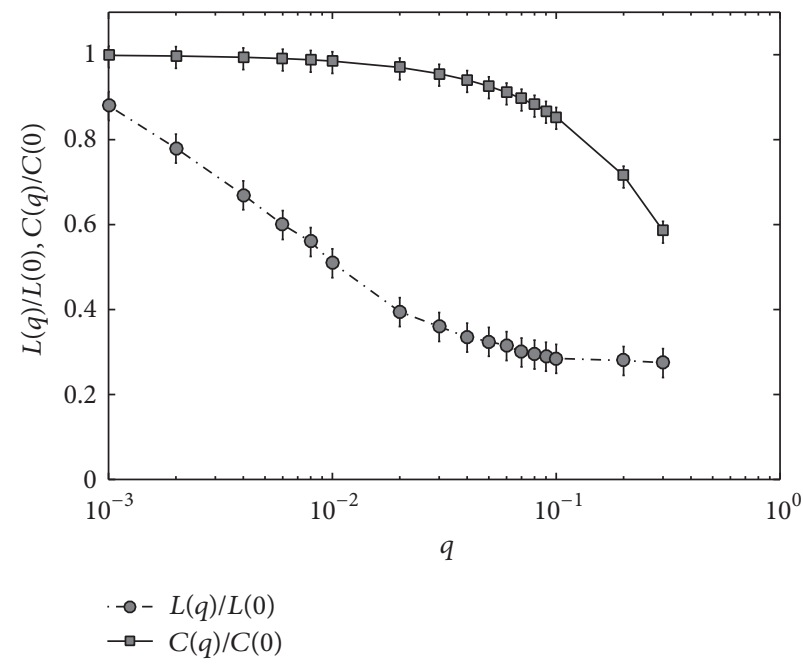

(d)

Figure 3: Analysis of average path length and clustering coefficient under different establishing schemes of shortcuts. (a) Scheme RC. (b) Scheme SC. (c) Scheme RCS. (d) Scheme SCS.

under different establishing schemes of shortcuts. $L(0)$ and $C(0)$ indicate the average path length and the clustering coefficient on the initial network without adding shortcut $(q=0)$, respectively. $L(q)$ and $C(q)$ indicate the average path length and the clustering coefficient with adding shortcut in accordance with the probability $q$. It is easy to find that, under 4 deployment schemes of shortcuts, the average path length of our model decreases much faster than the network clustering coefficient. Therefore, from the curve, it is easy to observe that, in our model no matter what shortcut placement scheme is, the network is able to have a small average path length while maintaining a relatively big clustering coefficient, which complies with the scale-free feature. This characteristic makes the topology generated by our model have a good connectivity no matter whether from the local or global perspective. But four schemes still present significant differences. As for scheme SCS, establishing shortcuts between the central cluster head and the sink node can reduce the average path length of the network rapidly at the beginning of the network evolution, which can ensure that only a few of shortcuts are required, and the hops from the sensor nodes to the sink node can be reduced significantly. In addition, there exists an upper limit of lifting effects of shortcuts $q_{t}=0.06$. When $q \geqslant q_{t}$, the lifting effects of adding more shortcuts tend to be not so apparent. At this moment, the average path length of the network $L\left(q \geqslant q_{t}\right)$ is only about $20 \%$ of the initial average path length $L(0)$. Obviously, the lower $q_{t}$ is, the less shortcuts the network would require.

From Figure 4, we can easily find that, compared with other three schemes, scheme SCS has the lowest $q_{t}$, which means the least number of shortcuts would be required in scheme SCS. And with the expanding size of the network, $q_{t}$ tends to be bigger, which means the larger the network is, the higher the proportion of shortcuts would be required. 


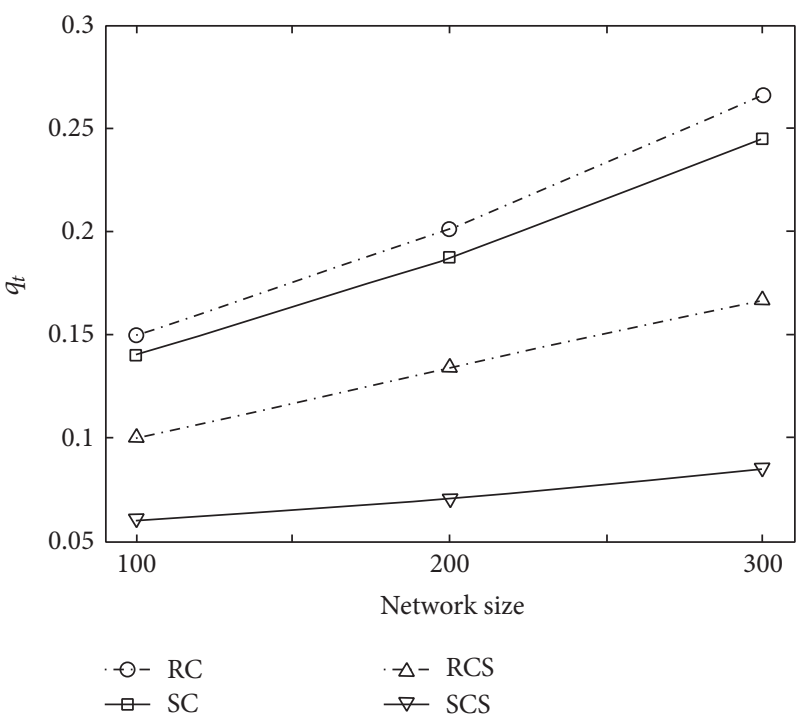

FIGURE 4: Comparison between practical degree distribution and theoretical distribution.

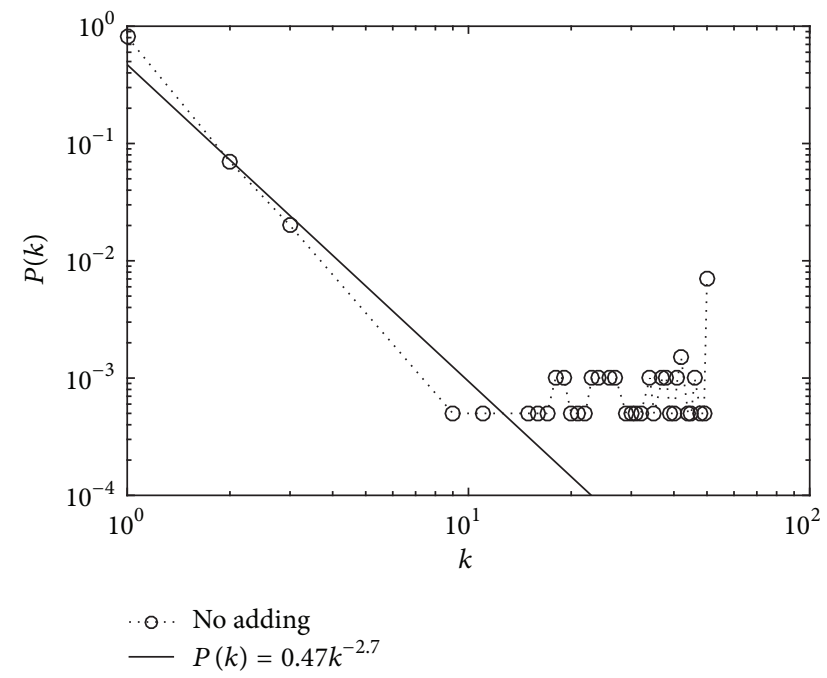

FIgURE 5: Comparison between practical degree distribution and theoretical distribution.

5.2. Analysis of Scale-Free Characteristics. In Figure 5, we conduct a comparison between simulation results and theoretical analysis from (24). Equation (24) would be $P(k)=$ $0.47 k^{-2.7}$ according to our simulation settings. From Figure 5, we can easily find that the general tends of both curves are close. As in our theoretical analysis the basic assumption is that the network scale is large enough which our simulation can hardly satisfy, there is a deviation between two results. With the increased size of the network, this difference would be reduced.

Figure 6 depicts the degree distribution of the network with different shortcut-adding probability (i.e., $q=0.01$ and $q=0.1$ ). It is not difficult to discover that the degree distribution of the network is closely in line with powerlaw distribution. In Figure 6(a), the total number of adding shortcuts is about $q \times N=10$. Apparently the impacts of shortcuts on degree distribution can be neglected. At this point, the degree of $86 \%$ sensor nodes is one and less than $1 \%$ of cluster heads achieve degree saturation. From the appended figure in Figure 6(a), it can be seen that when schemes SC and SCS are carried on, the maximum degree of cluster heads in the network increases to 51. By contrast, as for schemes RC and RCS, the maximum degree of cluster heads is 50 , which is still consistent with the network without adding shortcuts. As shown in Figure 6(b), the differences of degree distribution among various deployment schemes are more obvious. Since schemes SC and SCS are based on the principle of preferential selection, it is highly likely for shortcuts to be placed at cluster heads with ample power and many connections, which makes the proportion of "edge node" (i.e., node of which degree is one) the same as the network with adding-shortcuts probability $q=0.01$, still $86 \%$. But for schemes RC and RCS, the percentage of edge node in the network declines obviously, $76 \%$ and $74 \%$, respectively. From the appended figure in Figure 6(b), compared with the network with adding-shortcuts probability $q=0.01$, the maximum degree of cluster heads in schemes SC and SCS has increased to 60 and the proportion of central cluster heads (i.e., cluster head of which degree is more than 10) has also grown significantly. Overall, the impacts of schemes RC and RCS on network degree distribution is more obvious than that of schemes SC and SCS. This is because, in schemes RC and RCS, the probability of edge node obtaining connections is increasing with the rising of probability of shortcuts $q$, which makes their degree distributions more sensitive to the variation of shortcut probability $q$. On the contrary, in schemes SC and SCS the central cluster heads are always the major receivers of new connections no matter what shortcut probability $q$ is, which limits the impacts of adding shortcuts on their degree distributions.

5.3. Analysis of Energy Consumption. With reference to the classic first-order radio energy consumption model of wireless communication [33], the simulation setting of energy consumption in our model is as follows:

(1) Considering the initial energy, the unit is joule (J).

(2) Message can be generated in either cluster heads or regular sensor nodes randomly and message generation probability for each time step $P_{M}=0.05$. The size of unit message is 128 bytes and energy consumption of sending and receiving for each byte is the same, both $E_{s / r}=1 \times 10^{-4} \mathrm{~J}$. Thus, the energy consumption of sending and receiving per unit message is equal to $0.128 \mathrm{~J}$.

(3) At each time step, energy consumption of one sensor node for monitoring $E_{w}=0.032 \mathrm{~J}$.

(4) When message is generated, it will be delivered to the sink node by the Dijkstra shortest path. If more than one shortest path exists, we only choose one from them as transferring route.

(5) If the energy of sensor node runs out or there is no effective link connecting between the sensor node 


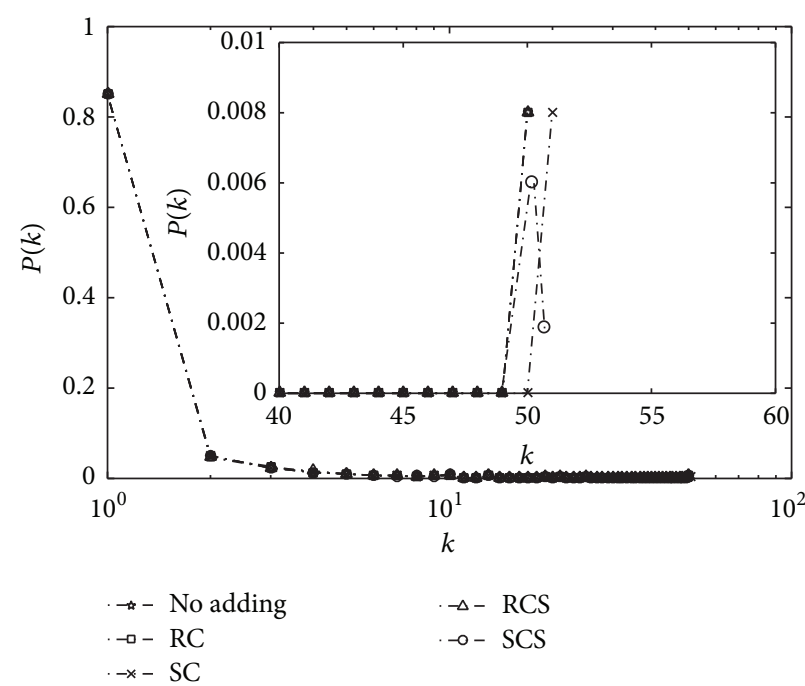

(a)

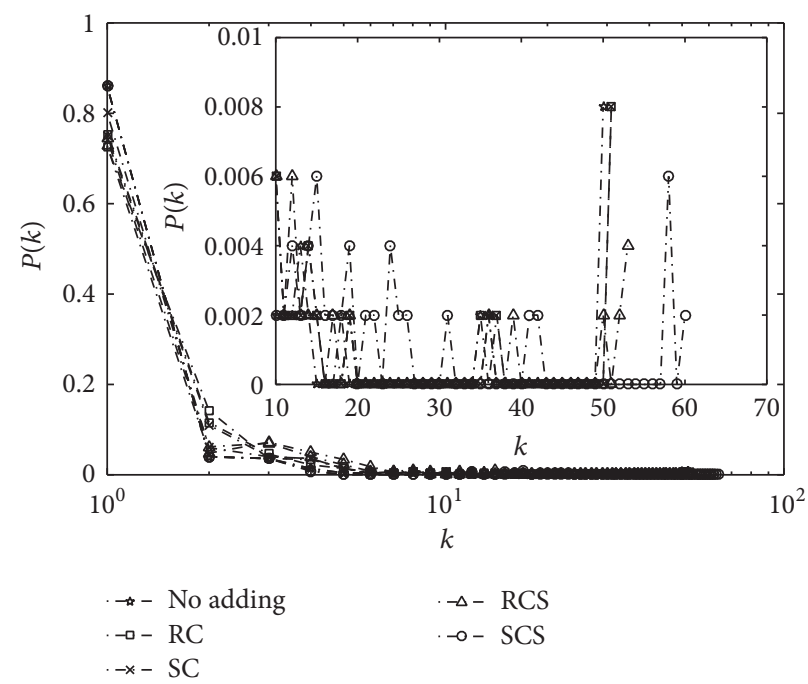

(b)

FIGURE 6: Degree distribution of the network with $q=0.01$ and $q=0.1$. (a) $q=0.01$. (b) $q=0.1$.

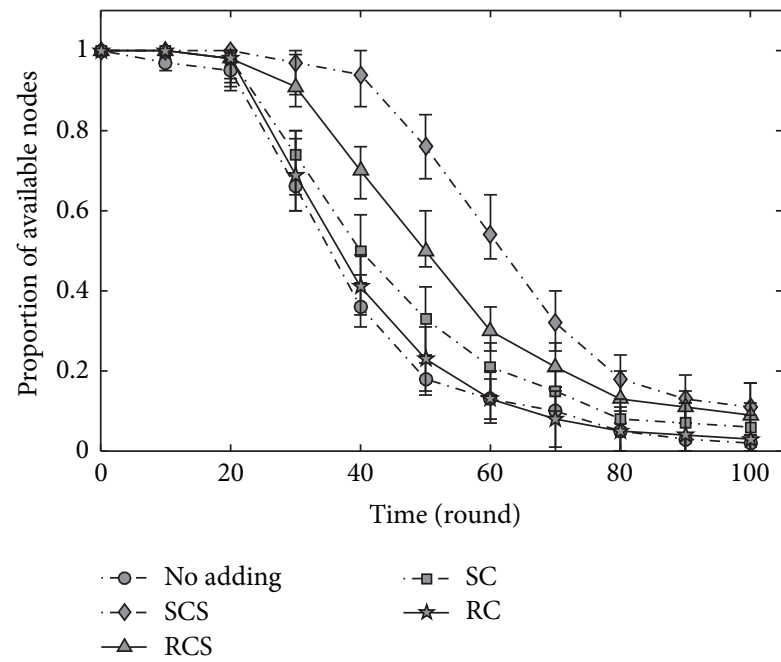

(a)

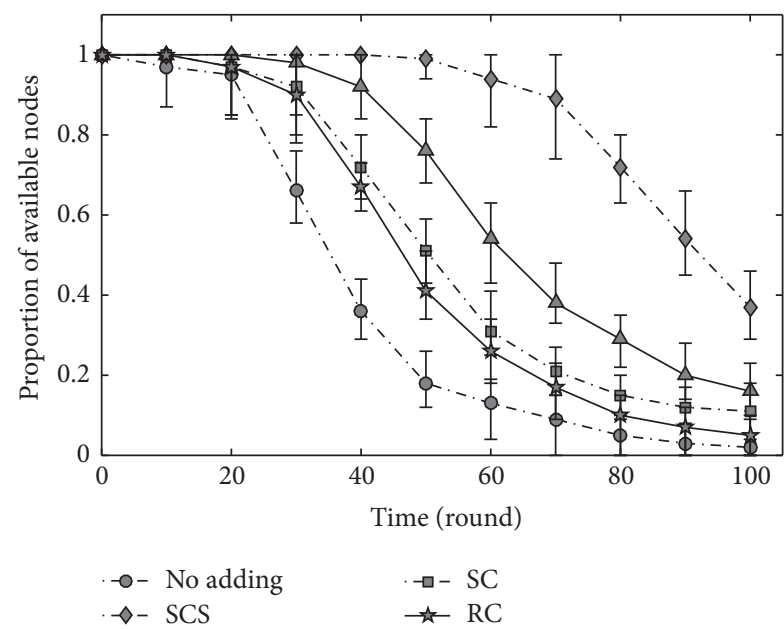

(b)

FIGURE 7: Energy consumption of the network with $q=0.01$ and $q=0.1$. (a) $q=0.01$. (b) $q=0.1$.

and the sink node, we reasonably consider it falling into "failure" state and this state is not reversible. For failure sensor node, it can be equivalent to "removing it from the existing network topology."

Figure 7 depicts the network performance in terms of energy consumption with shortcut-adding probabilities $q=$ 0.01 and $q=0.1$. According to the various placement schemes, adding shortcuts to the network can significantly improve the energy performance. As is shown in Figure 7(a), when $q=0.01$, scheme SCS performs best. When the network runs into 50th time step, about $80 \%$ of sensor nodes can still operate well. By contrast, for the network without adding shortcuts, only less than $20 \%$ of the sensor networks have enough energy to maintain working, which can make the fact that the entire network is paralyzed reasonably considered.
Through comparison analysis with Figure 3, it can be noticed that the energy consumption is closely related to the average path length. Apparently, in scheme SCS the shortcut is built between central cluster heads and the sink node, which makes the hops of message delivery decrease rapidly, thus improving the energy efficiency of the network. By contrast, in scheme RC the shortcuts are randomly deployed between cluster heads, and the decline of average path length is not so evident, which makes the lifting effects of shortcuts on energy conservation so limited. But it is worthy to note that although in scheme RCS shortcuts are randomly deployed between cluster heads and the sink node, it is still far superior to scheme SC based on preferential selection. This is because, through building shortcuts between cluster heads and the sink node, the cluster heads at the endpoints of the shortcuts 


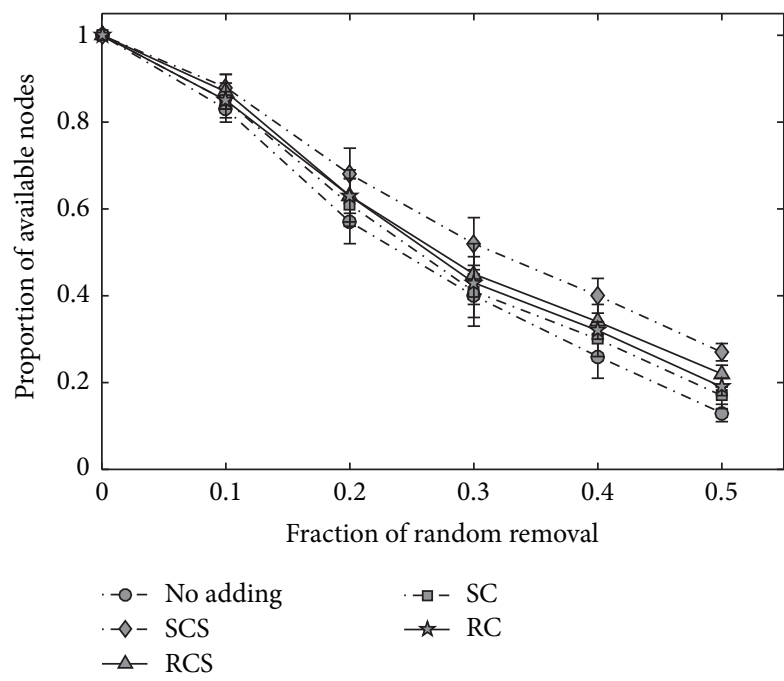

(a)

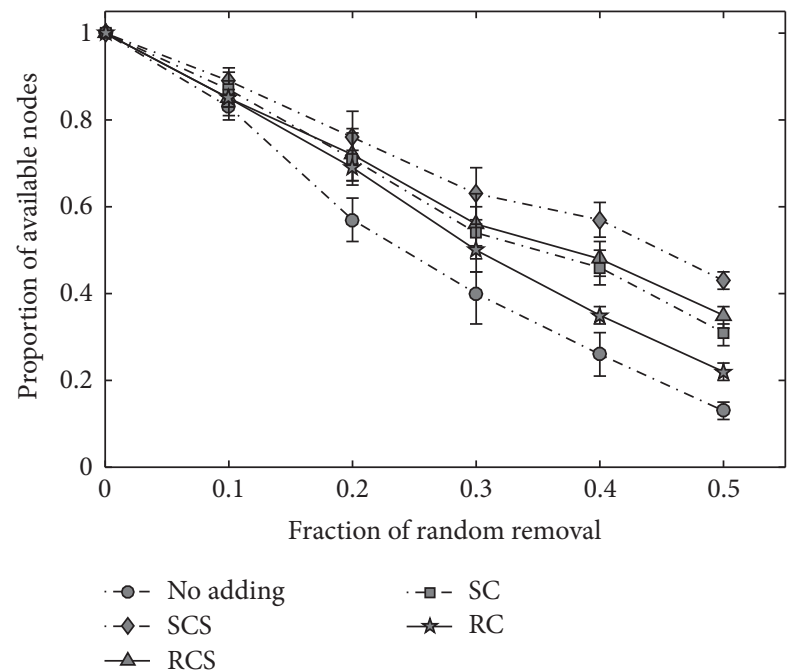

(b)

FIGURE 8: Error tolerance of the network with $q=0.01$ and $q=0.1$. (a) $q=0.01$. (b) $q=0.1$.

are able to deliver message to the sink directly without passing by other relaying node, which can reduce the communication load of the cluster heads around the sink node, especially the cluster heads with one hop from the sink. To be more specific, the sensor nodes nearby the sink run out of energy much faster than other nodes due to taking more message-relaying tasks. We generally define this phenomenon as "energy hole" effect [34]. And the shortcuts in scheme RCS can significantly relieve this effect by building direct link between sensor nodes and the sink node. But for scheme SC, although the distance from the sensor node to the sink decreases significantly and distribution of energy load is more balanced, most of messages still need to pass by the cluster heads nearby the sink and the overall load of these nodes is not reduced. As is shown in Figure 7(b), with the rising of probability $q$, the energy consumption has been further improved and general tendency has not changed. Compared with other three kinds of placement schemes, the advantage of scheme SCS is more apparent.

5.4. Analysis of Network Invulnerability. The invulnerability of WSNs measures the ability of the network providing sustainable and stable service under uncertain environments. According to the differences of failure models, the invulnerability can be subcategorized into error tolerance and intrusion tolerance [35]. Error tolerance means the invulnerability of the network against random damage. In the real applications of WSNs, random damage exists everywhere. Hardware or software malfunction and node/link breakdown caused by nature disasters (e.g., rainstorm, earthquake) are the most common instances of random damage. Generally, we choose the availability of network topology under random attack as metric to measure the error tolerance performance of the network. Intrusion tolerance means the invulnerability of the network against intended damage. Compared with random damage, intended damage means the attacker will destroy the sensor nodes according to the importance level of the nodes in the network. Obviously, the more important the node is, the higher the probability it would have to be attacked. In most of application scenarios, hacker invasion or malicious destruction is the most common form of intended damage. Since in our model degree is the most important factor that can determine how many new connections the sensor node can acquire, here we still choose degree as the indicator to determine the importance level of a node in the network. For intrusion tolerance, we choose the availability of network topology under maximum degree attack as metric to measure the intrusion tolerance performance of the network. This means the attacker will destroy the sensor node from the highest degree to lowest degree. By referencing [28], we use the proportion of available nodes to represent the availability of the network topology. After the network suffers from damage, if the sensor nodes can still maintain at least one effective link towards the sink node, we can consider that the node is still working.

5.4.1. Error Tolerance. Figure 8 depicts the error tolerance of the network with adding-shortcuts probabilities $q=$ 0.01 and $q=0.1$. As is shown in Figure 8(a), through establishing shortcuts, the error tolerance is improved to a certain degree. Among four deployment schemes, the lifting effects of schemes RCS and SCS are the most obvious. When $50 \%$ of the sensor nodes are removed from the network, we still have that $30 \%$ and $27 \%$ of sensor nodes can maintain at least one connection with the sink. As is shown in Figure 8(b), with the increasing of adding-shortcuts probability $q$, the network performance in terms of error tolerance is improved a lot. As for scheme SCS, even though $50 \%$ of sensor nodes are removed, we still have more than $47 \%$ of the nodes operating well, which means the connectivity of the remaining node is little affected by node removal.

5.4.2. Intrusion Tolerance. Figure 9 demonstrates the intrusion tolerance of the network with shortcuts-adding 


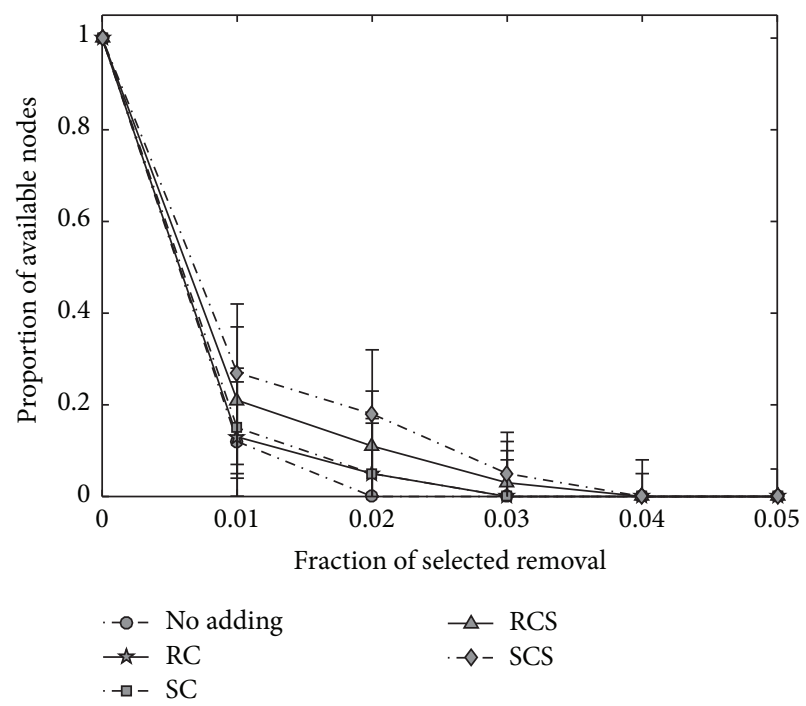

(a)

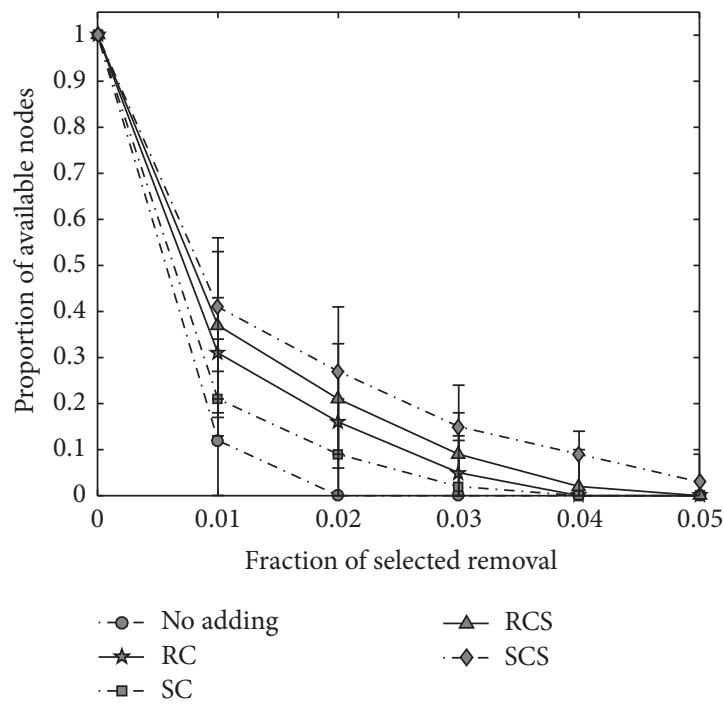

(b)

FIGURE 9: Intrusion tolerance of the network with $q=0.01$ and $q=0.1$. (a) $q=0.01$. (b) $q=0.1$.

probabilities $q=0.01$ and $q=0.1$. Compared with the lifting effects of shortcuts on error tolerance, the improvement brought by shortcuts on intrusion tolerance is not so evident. For the network without adding shortcuts, removing $1 \%$ of nodes from highest degree to lowest degree can give rise to the paralysis of the network. At this point, only less than $12 \%$ of sensor nodes can operate well. As mentioned before, for scale-free WSNs, only a few number of central nodes (i.e., usually high-degree nodes) account for a vast majority of connections. If these sensor nodes fall into "failure" state, the remaining sensor nodes will also be damaged due to the link interruption towards to the sink.

As shown in Figure 9(a), shortcuts are added to the network with probability $q=0.01$. Intrusion tolerance of the network has been improved to some extent but is not so obvious. Among four placement schemes, the scheme SCS has the optimal performance. After $1 \%$ of central node removing from the network, more than $27 \%$ of sensor nodes can still keep working. The lifting effects of scheme RCS come second. But for schemes RC and SC, the improvement brought by shortcuts is not significant. This is because, despite the fact that introduction of scheme RC or SC can effectively improve the network connectivity, most of messages still need to be relayed via central nodes. When the network encounters the intended attack, these kinds of nodes have highest priority to be attacked, thus leading to the breakdown of the entire network. As shown in Figure 9(b), when $q$ rises to 0.1, the intrusion tolerance is further improved, but the performance is still far from satisfactory. As far as scheme SCS is concerned, if $1 \%$ of central nodes are deleted from the network, the proportion of survival sensor nodes rises from $27 \%$ to $41 \%$.

\section{Conclusions and Future Works}

Scale-free network and small-world network provide new inspirations for building a highly effective WSNs topology. In this paper, we have presented a new WSNs topology evolution model with both considerations of characteristics of scalefree and small-world networks. In our model, the generated topology is cluster-structured, which is more suitable for the real application scenarios. Aiming to prolong the lifetime of the network, energy sensitivity and maximum degree limitation are introduced into the model. Besides that, in order to construct small-world effects in WSNs, we introduce shortcuts into the network during the process of evolution. The theoretical and simulation results have successfully proven the following:

(1) The degree distribution of our model is featured by power-law. Besides that, the generated network topology can have small average path length while maintaining big clustering coefficient. Thus, our model can guarantee that the generated network could have characteristics of scale-free network and small-world network at the same time, thus making the network maintain superior error tolerance and have better energy efficiency and intrusion tolerance.

(2) Different deployment schemes of shortcuts can produce different effects. Due to this reason, in this paper we research four placement schemes (i.e., RC, SC, RCS, and SCS). In general, the schemes that construct shortcuts between cluster heads and the sink node have better promoted effects than the scheme that builds shortcuts between pairs of cluster heads. The schemes based on the preferential principle are superior to the schemes based on the random principle. Therefore, the scheme SCS performs best among four proposed schemes as it only selects central cluster heads and the sink node as the endpoints of the shortcuts.

But it is still worthy to note that, despite the fact that building shortcuts between cluster heads and the sink can 
maximize the lifting effects of the shortcuts, this scheme might encounter some realistic challenges. In many application scenarios, the sink node can hardly provide enough number of wired interfaces for shortcuts. In addition, compared with schemes RC and SC, the scheme like RCS or SCS has stricter requirements for the processing speed, cache space, and other hardware configurations. Therefore, it is still necessary for us to choose a cost-effective placement scheme according to the application scenarios.

In the future works, there are some research directions worthy for further investigation in our opinion:

(1) In this paper, we only assume that the shortcuts are not constrained. In other words, the shortcuts can be placed anywhere we want. But in the practical scenarios, the layout of shortcuts is usually restricted by many factors (e.g., wire length, node/wire density, and geographical obstructions). Therefore, in order to guarantee that the generated network topology has strong suitability in the real environment, designing a deployment scheme of shortcuts with consideration of multiple constraints is worthy of exploring next.

(2) In this paper, we use the wired cables to play the role of shortcuts. In some scenarios, wired cables do have some advantages (e.g., easy-deployment and lowcost), but their flexibility is far from satisfactory as they cannot be changed dynamically once deployed. Therefore, to avoid this drawback, the idea of using mobile robots can be useful. Robots would move back and forth to relay the data from source to the destination and the mobility trace of robots can be seen as shortcut in another way.

(3) Existing WSNs routings cannot be applied to our topology directly due to the existence of shortcuts. Therefore, it is necessary for us to develop a matching routing mechanism for our topology.

\section{Notations}

$m_{0}$ : Number of nodes before beginning the evolution of the network

$e_{0}$ : Number of edges before beginning the evolution of the network

$N(t)$ : The sum of cluster heads at time $t$

$k_{i}$ : $\quad$ Degree of node $i$ (i.e., number of links connected to node $i$ )

$k_{i, t}$ : Degree of node $i$ at time $t$

$k_{\max }:$ Maximum number of connections that a cluster head could have

$\langle k(t)\rangle$ : Average degree of the cluster heads in the network

$p$ : $\quad$ Proportion of cluster heads in the network

$q$ : $\quad$ Probability of adding shortcuts at every time step

$E_{i}$ : $\quad$ Remaining energy of node $i$

$P_{s}(i)$ : Probability of a newly coming node that will be connected to node $i$

$P_{a}(i)$ : Probability of a newly coming shortcut that will be connected to node $i$
$\bar{E}$ : Average remaining energy of the nodes in the network

$E_{\max }:$ Maximum energy that a node could have

$\rho(E)$ : Probability density of remaining energy of the nodes in the interval $\left[0, E_{\max }\right]$

$S(t)$ : Existing node number at time $t$

$S: \quad$ Expected node number.

\section{Conflicts of Interest}

The authors declare that there are no conflicts of interest regarding the publication of this paper.

\section{Acknowledgments}

This work has been financially supported by the National Natural Science Foundation of China (61571336), Science and Technology Project of Henan province, China (172102210081), and the joint bilateral Italy/China project "Smart Personal Mobility Systems for Human Disabilities in Future Smart Cities" (China-side Project ID: 2015DFG12210; Italy-side Project ID: CN13MO7).

\section{References}

[1] W.-F. Li and X.-W. Fu, "Survey on invulnerability of wireless sensor networks," Jisuanji Xuebao/Chinese Journal of Computers, vol. 38, no. 3, pp. 625-647, 2015.

[2] G. Fortino, A. Guerrieri, G. M. P. O’Hare, and A. Ruzzelli, "A flexible building management framework based on wireless sensor and actuator networks," Journal of Network and Computer Applications, vol. 35, no. 6, pp. 1934-1952, 2012.

[3] R. Meseguer, C. Molina, S. F. Ochoa, and R. Santos, "Energyaware topology control strategy for human-centric wireless sensor networks," Sensors (Switzerland), vol. 14, no. 2, pp. 26192643, 2014.

[4] L. Fichera, F. Messina, G. Pappalardo, and C. Santoro, "A Python framework for programming autonomous robots using a declarative approach," Science of Computer Programming, vol. 139, pp. 36-55, 2017.

[5] A. Nazi, M. Raj, M. Di Francesco, P. Ghosh, and S. K. Das, "Deployment of robust wireless sensor networks using gene regulatory networks: An isomorphism-based approach," Pervasive and Mobile Computing, vol. 13, pp. 246-257, 2014.

[6] M. De Benedetti, F. Messina, G. Pappalardo, C. Santoro et al., "JarvSis: a distributed scheduler for IoT applications," Cluster Computing, vol. 20, no. 2, pp. 1-16, 2017.

[7] S. H. Strogatz, "Exploring complex networks," Nature, vol. 410, no. 6825 , pp. 268-276, 2001.

[8] B. Karrer and M. E. J. Newman, "Competing epidemics on complex networks," Physical Review E, vol. 84, no. 3, Article ID 036106, 2011.

[9] A. L. Barabási, R. Albert, and H. Jeong, "Mean-field theory for scale-free random networks," Physica A: Statistical Mechanics and its Applications, vol. 272, no. 1, pp. 173-187, 1999.

[10] R. Pastor-Satorras and A. Vespignani, "Epidemic spreading in scale-free networks," Physical Review Letters, vol. 86, no. 14, pp. 3200-3203, 2001. 
[11] M. Rubinov and O. Sporns, "Complex network measures of brain connectivity: Uses and interpretations," NeuroImage, vol. 52, no. 3, pp. 1059-1069, 2010.

[12] D. J. Watts and S. H. Strogatz, "Collective dynamics of "smallworld” networks," Nature, vol. 393, no. 6684, pp. 440-442, 1998.

[13] L.-J. Chen, D.-X. Chen, L. Xie, and J.-N. Cao, "Evolution of wireless sensor network," in Proceedings of the IEEE Wireless Communications and Networking Conference (WCNC '07), vol. 556, pp. 3005-3009, March 2007.

[14] H. Zhu, H. Luo, H. Peng, L. Li, and Q. Luo, "Complex networksbased energy-efficient evolution model for wireless sensor networks," Chaos, Solitons and Fractals, vol. 41, no. 4, pp. 18281835, 2009.

[15] S. Li, L. Li, and Y. Yang, "A local-world heterogeneous model of wireless sensor networks with node and link diversity," Physica A: Statistical Mechanics and Its Applications, vol. 390, no. 6, pp. 1182-1191, 2011.

[16] N. Jiang, H. Chen, and X. Xiao, "A local world evolving model for energy-constrained wireless sensor networks," International Journal of Distributed Sensor Networks, vol. 2012, Article ID 542389, 2012.

[17] X. Qi, S. Ma, and G. Zheng, "Topology evolution of wireless sensor networks based on adaptive free-scale networks," Journal of Information and Computational Science, vol. 8, no. 3, pp. 467475, 2011.

[18] G. Zheng, S. Liu, and X. Qi, "Scale-free topology evolution for wireless sensor networks with reconstruction mechanism," Computers and Electrical Engineering, vol. 38, no. 3, pp. 643-651, 2012.

[19] L.-J. Chen, M. Liu, D.-X. Chen, and L. Xie, “Topology evolution of wireless sensor networks among cluster heads by random walkers," Jisuanji Xuebao/Chinese Journal of Computers, vol. 32, no. 1, pp. 69-76, 2009.

[20] Y. Q. Wang and X. Y. Yang, "A random walk evolution model of wireless sensor networks and virus spreading," Chinese Physics B, vol. 22, no. 1, Article ID 010509, 2013.

[21] A. Helmy, "Small worlds in wireless networks," IEEE Communications Letters, vol. 7, no. 10, pp. 490-492, 2003.

[22] R. Chitradurga and A. Helmy, "Analysis of Wired Short Cuts in Wireless Sensor Networks," in Proceedings of the The IEEE/ACS International Conference on Pervasive Services, pp. 167-176, Beirut, Lebanon, 2004.

[23] G. Sharma and R. Mazumdar, "Hybrid sensor networks: a small world," in Proceedings of the 6th ACM International Symposium on Mobile Ad Hoc Networking and Computing, pp. 366-377, May 2005.

[24] K. A. Hawick and H. A. James, "Small-world effects in wireless agent sensor networks," International Journal of Wireless and Mobile Computing, vol. 4, no. 3, pp. 155-164, 2010.

[25] S. Toyonaga, D. Kominami, and M. Murata, "Virtual wireless sensor networks: Adaptive brain-inspired configuration for internet of things applications," Sensors (Switzerland), vol. 16, no. 8, article no. 1323, 2016.

[26] D. L. Guidoni, R. A. F. Mini, and A. A. F. Loureiro, "On the design of heterogeneous sensor networks based on small world concepts," in Proceedings of the 11th ACM International Conference on Modeling, Analysis, and Simulation of Wireless and Mobile Systems, MSWiM'08, pp. 309-314, can, October 2008.

[27] D. L. Guidoni, R. A. F. Mini, and A. A. F. Loureiro, "On the design of resilient heterogeneous wireless sensor networks based on small world concepts," Computer Networks, vol. 54, no. 8, pp. 1266-1281, 2010.

[28] X. Fu, W. Li, and G. Fortino, "Empowering the invulnerability of wireless sensor networks through super wires and super nodes," in Proceedings of the 13th IEEE/ACM International Symposium on Cluster, Cloud, and Grid Computing, CCGrid 2013, pp. 561568, nld, May 2013.

[29] L. Lei, Y. Kuang, X. S. Shen, K. Yang, J. Qiao, and Z. Zhong, "Optimal reliability in energy harvesting industrial wireless sensor networks," IEEE Transactions on Wireless Communications, vol. 15, no. 8, pp. 5399-5413, 2016.

[30] S. N. Dorogovtsev, J. F. F. Mendes, and A. N. Samukhin, "Structure of growing networks with preferential linking," Physical Review Letters, vol. 85, no. 21, pp. 4633-4636, 2000.

[31] P. L. Krapivsky, S. Redner, and F. Leyvraz, "Connectivity of growing random networks," Physical Review Letters, vol. 85, no. 21, pp. 4629-4632, 2000.

[32] N. Sarshar and V. Roychowdhury, "Scale-free and stable structures in complex ad hoc networks," Physical Review E-Statistical, Nonlinear, and Soft Matter Physics, vol. 69, no. 2, Article ID 026101, pp. 1-6, 2004.

[33] W. R. Heinzelman, A. Chandrakasan, and H. Balakrishnan, "Energy-efficient communication protocol for wireless microsensor networks," in Proceedings of the 33rd Annual Hawaii International Conference on System Siences (HICSS '00), vol. 2, IEEE, January 2000.

[34] X. Wu, G. Chen, and S. K. Das, "Avoiding energy holes in wireless sensor networks with nonuniform node distribution," IEEE Transactions on Parallel and Distributed Systems, vol. 19, no. 5, pp. 710-720, 2008.

[35] R. Albert, H. Jeong, and A.-L. Barabási, "Error and attack tolerance of complex networks," Nature, vol. 406, no. 6794, pp. $378-382,2000$. 


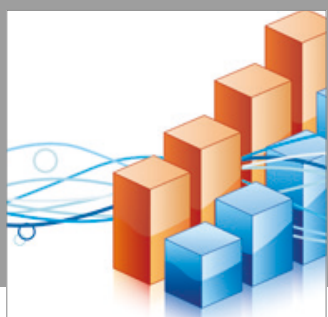

Advances in

Operations Research

vatersals

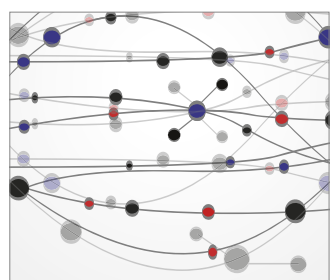

\section{The Scientific} World Journal
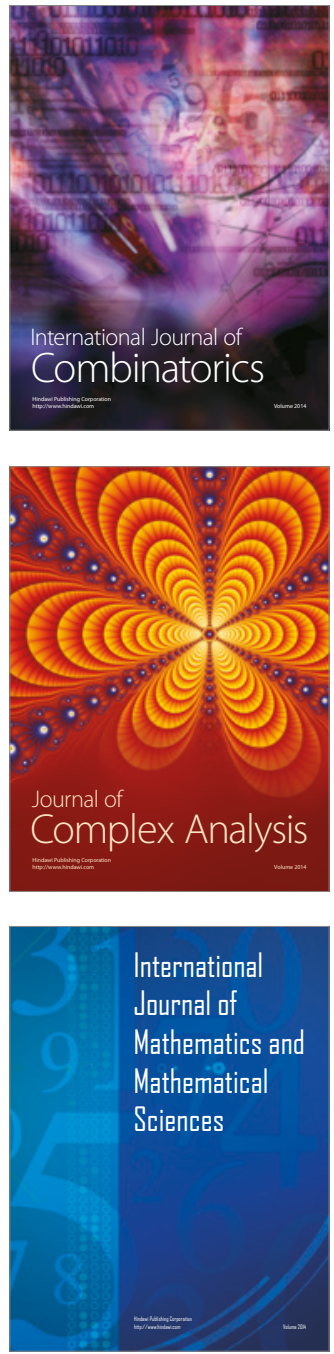
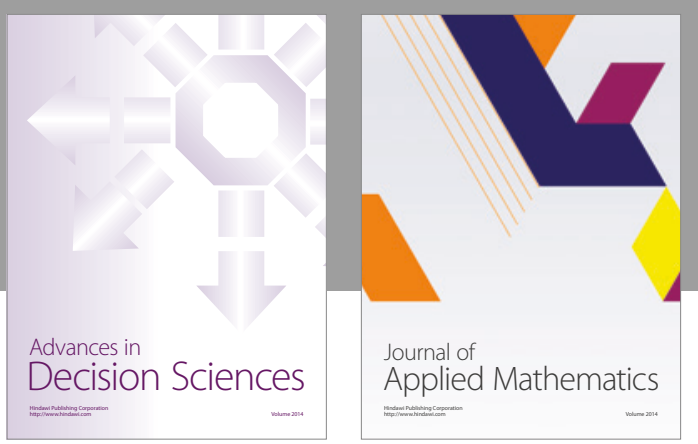

Algebra

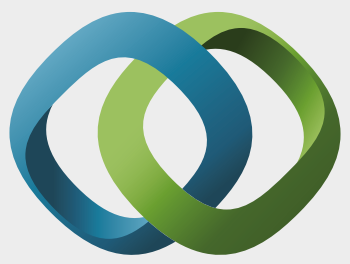

\section{Hindawi}

Submit your manuscripts at

https://www.hindawi.com
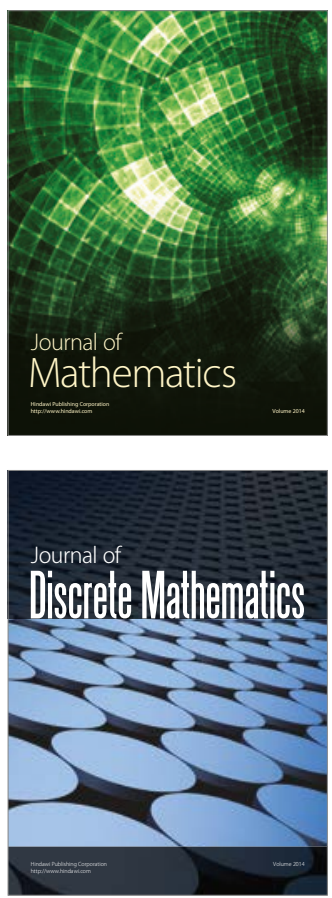

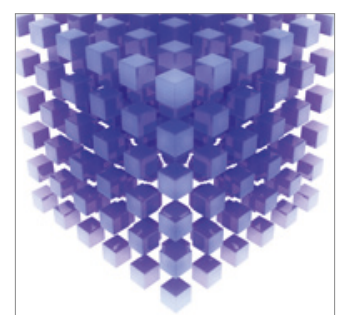

Mathematical Problems in Engineering
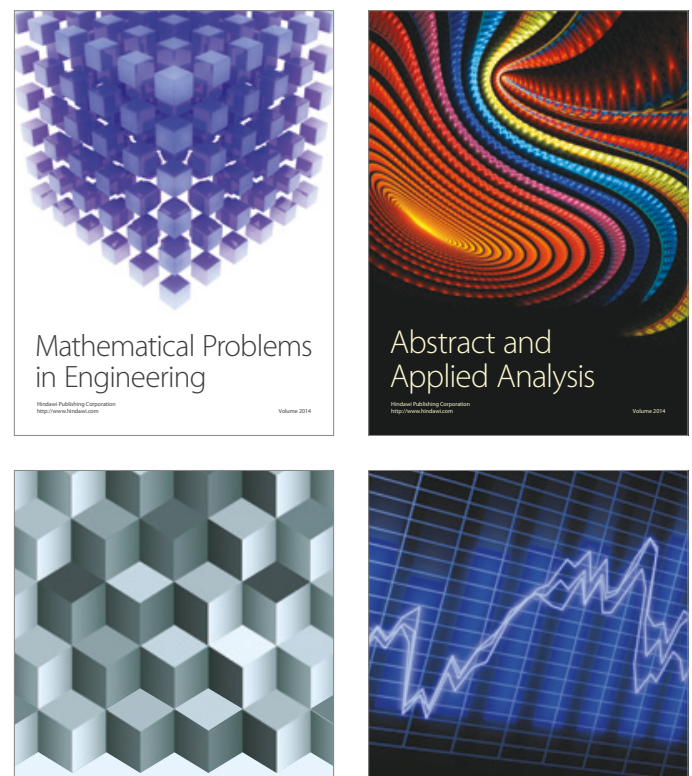

Journal of

Function Spaces

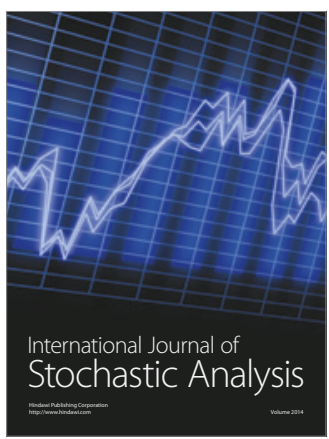

Probability and Statistics
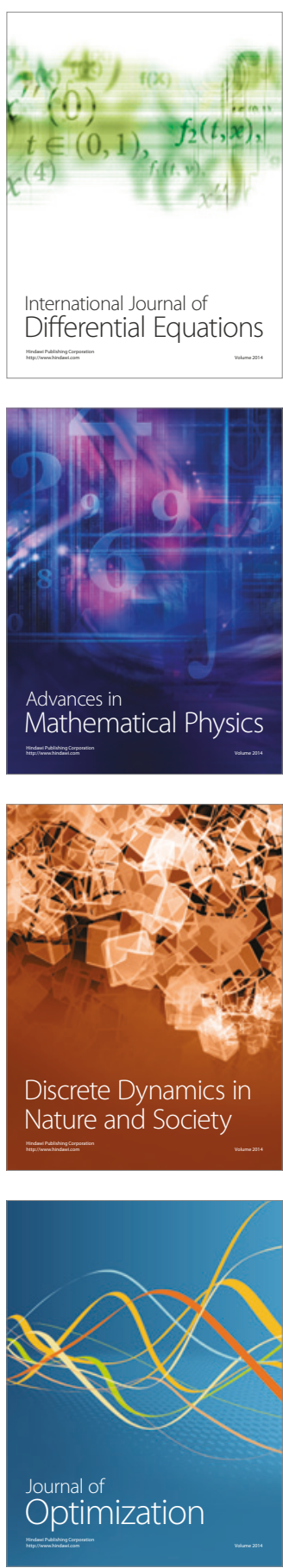